\title{
Existence and uniqueness of solutions for system of Hilfer-Hadamard sequential fractional differential equations with two point boundary conditions
}

\author{
Warissara Saengthong ${ }^{1}$, Ekkarath Thailert ${ }^{1,2^{*}}$ (D) and Sotiris K. Ntouyas ${ }^{3,4}$
}

"Correspondence:

ekkaratht@nu.ac.th

1 Department of Mathematics,

Faculty of Science, Naresuan

University, Phitsanulok, Thailand

${ }^{2}$ Research Center for Academic

Excellence in Mathematics,

Naresuan University, Phitsanulok,

Thailand

Full list of author information is

available at the end of the article

\section{勿 Springer}

\begin{abstract}
In this paper, we study existence and uniqueness of solutions for a system of Hilfer-Hadamard sequential fractional differential equations via standard fixed point theorems. The existence is proved by using the Leray-Schauder alternative, while the existence and uniqueness by the Banach contraction mapping principle. Illustrative examples are also discussed.
\end{abstract}

MSC: $34 \mathrm{~A} 08 ; 34 \mathrm{~B} 10$

Keywords: Hilfer-Hadamard fractional differential equations; Boundary conditions; Fixed point

\section{Introduction}

Fractional differential equations have been applied in many fields such as physics, chemistry, biology, engineering, and so on. Fractional differential equations have several kinds of fractional derivatives, such as Riemann-Liouville fractional derivative, Caputo fractional derivative, Grunwald-Letnikov fractional derivative, Hadamard fractional derivative, etc. The reader interested in the subject of fractional calculus is referred to the books by Kilbas et al. [1], Podlubny [2], Samko et al. [3], Miller and Ross [4], and Diethelm [5]. A generalization of derivatives of both Riemann-Liouville and Caputo was given by Hilfer in [6] when he studied fractional time evolution in physical phenomena. He named it generalized fractional derivative of order $\alpha \in(0,1)$ and type $\beta \in[0,1]$ which can be reduced to the Riemann-Liouville and Caputo fractional derivatives when $\beta=0$ and $\beta=1$, respectively. Many authors call it the Hilfer fractional derivative. Such derivative interpolates between the Riemann-Liouville and Caputo derivatives. For other current definitions of fractional derivatives, see [7-11].

Fractional-order boundary value problems have been extensively studied by many researchers. In particular, coupled systems of fractional-order differential equations have attracted special attention in view of their occurrence in the mathematical modeling of physical phenomena like chaos synchronization [12], anomalous diffusion [13], ecological

(c) The Author(s) 2019. This article is licensed under a Creative Commons Attribution 4.0 International License, which permits use sharing, adaptation, distribution and reproduction in any medium or format, as long as you give appropriate credit to the original author(s) and the source, provide a link to the Creative Commons licence, and indicate if changes were made. The images or other third party material in this article are included in the article's Creative Commons licence, unless indicated otherwise in a credit line to the material. If material is not included in the article's Creative Commons licence and your intended use is not permitted by statutory regulation or exceeds the permitted use, you will need to obtain permission directly from the copyright holder. To view a copy of this licence, visit http://creativecommons.org/licenses/by/4.0/. 
effects [14], disease models [15], etc. Additionally, fixed point theory can be used to develop the existence theory for the coupled systems of fractional differential equations. For some recent theoretical results on coupled systems of fractional-order differential equations, for example, see [16-30].

Alsaedi et al. [23] studied the existence of solutions for a Riemann-Liouville coupled system of nonlinear fractional integro-differential equations given by

$$
\begin{cases}D^{\alpha} u(t)=f\left(t, u(t), v(t),\left(\phi_{1} u\right)(t),\left(\psi_{1} v\right)(t)\right), & t \in[0, T], \\ D^{\beta} v(t)=g\left(t, u(t), v(t),\left(\phi_{2} u\right)(t),\left(\psi_{2} v\right)(t)\right), & 1<\alpha, \beta \leq 2,\end{cases}
$$

subject to the coupled Riemann-Liouville integro-differential boundary conditions

$$
\left\{\begin{array}{lll}
D^{\alpha-2} u\left(0^{+}\right)=0, & D^{\alpha-1} u\left(0^{+}\right)=v I^{\alpha-1} v(\eta), & 0<\eta<T, \\
D^{\beta-2} v\left(0^{+}\right)=0, & D^{\beta-1} v\left(0^{+}\right)=\mu I^{\beta-1} u(\sigma), & 0<\sigma<T,
\end{array}\right.
$$

where $D^{(\cdot)}, I^{(\cdot)}$ denote the Riemann-Liouville derivatives and integral of fractional order $(\cdot)$, respectively, $f, g:[0, T] \times \mathbb{R}^{4} \rightarrow \mathbb{R}$ are given continuous functions, $\nu, \mu$ are real constants, and

$$
\begin{array}{ll}
\left(\phi_{1} u\right)(t)=\int_{0}^{t} \gamma_{1}(t, s) u(s) d s, & \left(\phi_{2} u\right)(t)=\int_{0}^{t} \gamma_{2}(t, s) u(s) d s \\
\left(\psi_{1} v\right)(t)=\int_{0}^{t} \delta_{1}(t, s) v(s) d s, & \left(\psi_{2} v\right)(t)=\int_{0}^{t} \delta_{2}(t, s) v(s) d s
\end{array}
$$

with $\gamma_{i}$ and $\delta_{i}(i=1,2)$ are continuous functions on $[0, T] \times[0, T]$.

Alsulami et al. [24] studied a new system of coupled Caputo type fractional differential equations

$$
\begin{cases}{ }^{c} D^{\alpha} u(t)=f(t, u(t), v(t)), & t \in[0, T], 1<\alpha \leq 2, \\ { }^{c} D^{\beta} v(t)=g(t, u(t), v(t)), & t \in[0, T], 1<\beta \leq 2,\end{cases}
$$

subject to the following non-separated coupled boundary conditions:

$$
\begin{cases}u(0)=\lambda_{1} v(T), & u^{\prime}(0)=\lambda_{2} v^{\prime}(T), \\ v(0)=\mu_{1} u(T), & v^{\prime}(0)=\mu_{2} u^{\prime}(T),\end{cases}
$$

where ${ }^{c} D^{\alpha},{ }^{c} D^{\beta}$ denote the Caputo fractional derivatives of order $\alpha$ and $\beta$, respectively, $f, g:[0, T] \times \mathbb{R} \times \mathbb{R} \rightarrow \mathbb{R}$ are appropriately chosen functions and $\lambda_{i}, \mu_{i}, i=1,2$, are real constants with $\lambda_{i} \mu_{i} \neq 1, i=1,2$.

Ahmad et al. [25] studied the existence and uniqueness of solutions for the following boundary value problem of nonlinear Caputo sequential fractional differential equations:

$$
\begin{cases}\left({ }^{c} D^{\alpha}+k_{1}{ }^{c} D^{\alpha-1}\right) u(t)=f(t, u(t), v(t)), & 1<\alpha \leq 2, t \in(0, T), \\ \left({ }^{c} D^{\beta}+k_{2}{ }^{c} D^{\beta-1}\right) v(t)=g(t, u(t), v(t)), & 1<\beta \leq 2, t \in(0, T),\end{cases}
$$


supplemented with coupled boundary conditions

$$
\begin{cases}u(0)=a_{1} v(T), & u^{\prime}(0)=a_{2} v^{\prime}(T) \\ v(0)=b_{1} u(T), & v^{\prime}(0)=b_{2} u^{\prime}(T)\end{cases}
$$

where ${ }^{c} D^{\alpha},{ }^{c} D^{\beta}$ denote the Caputo fractional derivatives of order $\alpha$ and $\beta$, respectively, $k_{1}, k_{2} \in \mathbb{R}_{+}, T>0, f, g:[0, T] \times \mathbb{R} \times \mathbb{R} \rightarrow \mathbb{R}$ are given continuous functions, and $a_{1}, a_{2}, b_{1}$, and $b_{2}$ are real constants with $a_{1} b_{1} \neq 1$ and $a_{2} b_{2} e^{-\left(k_{1} T+k_{2} T\right)} \neq 1$.

Aljoudi et al. [29] studied a coupled system of Hadamard type sequential fractional differential equations with coupled strip conditions given by

$$
\left\{\begin{array}{l}
\left(D^{q}+k D^{q-1}\right) u(t)=f\left(t, u(t), v(t), D^{\alpha} v(t)\right), \quad k>0,1<q \leq 2,0<\alpha<1, \\
\left(D^{p}+k D^{p-1}\right) v(t)=g\left(t, u(t), v(t), D^{\delta} u(t)\right), \quad 1<p \leq 2,0<\delta<1, \\
u(1)=0, \quad u(e)=I^{\gamma} v(\eta)=\frac{1}{\Gamma(\gamma)} \int_{1}^{\eta}\left(\log \frac{\eta}{s}\right)^{\gamma-1} \frac{v(s)}{s} d s, \quad \gamma>0,1<\eta<e, \\
v(1)=0, \quad v(e)=I^{\beta} v(\zeta)=\frac{1}{\Gamma(\beta)} \int_{1}^{\zeta}\left(\log \frac{\zeta}{s}\right)^{\beta-1} \frac{u(s)}{s} d s, \quad \beta>0,1<\zeta<e,
\end{array}\right.
$$

where $D^{(\cdot)}$ and $I^{(\cdot)}$ denote the Hadamard fractional derivative and the Hadamard fractional integral, respectively, and $f, g:[1, e] \times \mathbb{R}^{3} \rightarrow \mathbb{R}$ are given continuous functions.

Motivated by the research going on in this direction, in this paper, we study existence and uniqueness of solutions for a new class of systems of Hilfer-Hadamard sequential fractional differential equations

$$
\begin{cases}\left({ }_{H} D_{1^{+}}^{\alpha_{1}, \beta_{1}}+k_{1 H} D_{1^{+}}^{\alpha_{1}-1, \beta_{1}}\right) u(t)=f(t, u(t), v(t)), & 1<\alpha_{1} \leq 2, t \in[1, e], \\ \left.{ }_{H} D_{1^{+}}^{\alpha_{2}, \beta_{2}}+k_{2 H} D_{1^{+}}^{\alpha_{2}-1, \beta_{2}}\right) v(t)=g(t, u(t), v(t)), & 1<\alpha_{2} \leq 2, t \in[1, e],\end{cases}
$$

with two-point boundary conditions

$$
\begin{cases}u(1)=0, & u(e)=A_{1}, \\ v(1)=0, & v(e)=A_{2},\end{cases}
$$

where ${ }_{H} D^{\alpha_{i}, \beta_{i}}$ is the Hilfer-Hadamard fractional derivative of order $\alpha_{i} \in(1,2]$ and type $\beta_{i} \in[0,1]$ for $i \in\{1,2\}, k_{1}, k_{2}, A_{1}, A_{2} \in \mathbb{R}_{+}$and $f, g:[1, e] \times \mathbb{R} \times \mathbb{R} \rightarrow \mathbb{R}$ are given continuous functions.

To the best of our knowledge, this is the first paper dealing with a system containing Hilfer-Hadamard fractional derivative of order $\alpha_{i} \in(1,2], i=1,2$. For some recent results on coupled systems of Hilfer-Hadamard fractional derivatives of order $\alpha_{i} \in(0,1], i=1,2$, we refer to [31, 32], and the references cited therein.

The paper is organized as follows. In Sect. 2, we present some preliminary concepts of fractional calculus. Section 3 contains the main results. The first result, Theorem 3.2, is proved by using the Leray-Schauder alternative and the second result of existence and uniqueness, Theorem 3.3, by the Banach contraction mapping principle. Finally, Sect. 4 provides some examples for the illustration of the main results. We emphasize that our results are new and contribute significantly to the topic addressed in this paper. 


\section{Preliminaries}

In this section, some basic definitions, lemmas, and theorems are mentioned.

Definition 2.1 (Hadamard fractional integral [1]) The Hadamard fractional integral of order $\alpha \in \mathbb{R}_{++}$for a function $f:[a, \infty) \rightarrow \mathbb{R}$ is defined as follows:

$$
{ }_{H} I_{a^{+}}^{\alpha} f(t)=\frac{1}{\Gamma(\alpha)} \int_{a}^{t}\left(\log \frac{t}{\tau}\right)^{\alpha-1} \frac{f(\tau)}{\tau} d \tau \quad(t>a)
$$

provided the integral exists, where $\log (\cdot)=\log _{e}(\cdot)$.

Definition 2.2 (Hadamard fractional derivative [1]) The Hadamard fractional derivative of order $\alpha>0$, applied to the function $f:[a, \infty) \rightarrow \mathbb{R}$, is defined as follows:

$$
{ }_{H} D_{a^{+}}^{\alpha} f(t)=\delta^{n}\left({ }_{H} I_{a^{+}}^{n-\alpha} f(t)\right), \quad n-1<\alpha<n, n=[\alpha]+1,
$$

where $\delta^{n}=\left(t \frac{d}{d t}\right)^{n}$ and $[\alpha]$ denotes the integer part of the real number $\alpha$.

Definition 2.3 (Hilfer-Hadamard fractional derivative [6,33]) Let $0<\alpha<1$ and $0 \leq$ $\beta \leq 1, f \in L^{1}(a, b)$. The Hilfer-Hadamard fractional derivative of order $\alpha$ and type $\beta$ of $f$ is defined as follows:

$$
\begin{aligned}
\left({ }_{H} D_{a^{+}}^{\alpha, \beta} f\right)(t) & =\left({ }_{H} I_{a^{+}}^{\beta(1-\alpha)} \delta_{H} I_{a^{+}}^{(1-\alpha)(1-\beta)} f\right)(t) \\
& =\left({ }_{H} I_{a^{+}}^{\beta(1-\alpha)} \delta_{H} I_{a^{+}}^{1-\gamma} f\right)(t) ; \quad \gamma=\alpha+\beta-\alpha \beta \\
& =\left({ }_{H} I_{a^{+}}^{\beta(1-\alpha)}{ }_{H} D_{a^{+}}^{\gamma} f\right)(t),
\end{aligned}
$$

where ${ }_{H} I_{a^{+}}^{(\cdot)}$ and ${ }_{H} D_{a^{+}}^{(\cdot)}$ are the Hadamard fractional integral and derivative defined by (3) and (4), respectively.

The Hilfer-Hadamard fractional derivative may be viewed as interpolating the Hadamard fractional derivative. Indeed, for $\beta=0$, this derivative reduces to the Hadamard fractional derivative.

Definition 2.4 (Hilfer-Hadamard fractional derivative [34]) Let $n-1<\alpha<n$ and $0 \leq$ $\beta \leq 1, f \in L^{1}(a, b)$. The Hilfer-Hadamard fractional derivative of order $\alpha$ and type $\beta$ of $f$ is defined as follows:

$$
\begin{aligned}
\left({ }_{H} D_{a^{+}}^{\alpha, \beta} f\right)(t) & =\left({ }_{H} I_{a^{+}}^{\beta(n-\alpha)} \delta^{n}{ }_{H} I_{a^{+}}^{(n-\alpha)(1-\beta)} f\right)(t) \\
& =\left({ }_{H} I_{a^{+}}^{\beta(n-\alpha)} \delta^{n}{ }_{H} I_{a^{+}}^{n-\gamma} f\right)(t) ; \quad \gamma=\alpha+n \beta-\alpha \beta \\
& =\left({ }_{H} I_{a^{+}}^{\beta(n-\alpha)}{ }_{H} D_{a^{+}}^{\gamma} f\right)(t),
\end{aligned}
$$

where ${ }_{H} I_{a^{+}}^{(\cdot)}$ and ${ }_{H} D_{a^{+}}^{(\cdot)}$ are the Hadamard fractional integral and derivative defined by (3) and (4), respectively.

We recommend some lemmas and theorems of the Hadamard fractional integral and derivative by Kilbas et al. [1]. 
Theorem 2.5 ([1, 35]) Let $\alpha>0, n=[\alpha]+1$, and $0<a<b<\infty$. If $f \in L^{1}(a, b)$ and $\left({ }_{H} I_{a+}^{n-\alpha} f\right)(t) \in A C_{\delta}^{n}[a, b]$, then

$$
\left({ }_{H} I_{a+}^{\alpha} D_{a+}^{\alpha} f\right)(t)=f(t)-\sum_{j=0}^{n-1} \frac{\left(\delta^{(n-j-1)}\left({ }_{H} I_{a+}^{n-\alpha} f\right)\right)(a)}{\Gamma(\alpha-j)}\left(\log \frac{t}{a}\right)^{\alpha-j-1}
$$

where $f(t) \in A C_{\delta}^{n}=\left\{f:[a, b] \rightarrow \mathbb{R}: \delta^{(n-1)} f(t) \in A C[a, b], \delta=t \frac{d}{d t}\right\}$

Theorem 2.6 ([33]) Let $\alpha>0,0 \leq \beta \leq 1, \gamma=\alpha+n \beta-\alpha \beta, n-1<\gamma \leq n, n=[\alpha]+1$, and $0<a<b<\infty$. Iff $\in L^{1}(a, b)$ and $\left({ }_{H} I_{a+}^{n-\gamma} f\right)(t) \in A C_{\delta}^{n}[a, b]$, then

$$
{ }_{H} I_{a+}^{\alpha}\left({ }_{H} D_{a+}^{\alpha, \beta} f\right)(t)={ }_{H} I_{a+}^{\gamma}\left({ }_{H} D_{a+}^{\gamma} f\right)(t)=f(t)-\sum_{j=0}^{n-1} \frac{\left(\delta^{(n-j-1)}\left({ }_{H} I_{a+}^{n-\gamma} f\right)\right)(a)}{\Gamma(\gamma-j)}\left(\log \frac{t}{a}\right)^{\gamma-j-1} .
$$

From this theorem, we notice that if $\beta=0$ the formulae reduce to the formulae in Theorem 2.5 .

We will use the following well-known fixed point theorems on Banach space for proving the existence and uniqueness of Hilfer-Hadamard fractional differential systems.

Theorem 2.7 (Leray-Schauder alternative [36]) Let $T: E \rightarrow$ E be a completely continuous operator (i.e., a continuous map $T$ restricted to any bounded set in $E$ is compact). Let $\varepsilon(T)=$ $\{x \in E: x=\lambda T(x), 0 \leq \lambda \leq 1\}$. Then either the set $\varepsilon(T)$ is unbounded or $T$ has at least one fixed point.

Theorem 2.8 (Banach fixed point theorem [37]) Let X be a Banach space, $D \subset X$ be closed, and $F: D \rightarrow D$ be a strict contraction, i.e., $\|F x-F y\| \leq k\|x-y\|$ for some $k \in(0,1)$ and all $x, y \in D$. Then $F$ has a fixed point in $D$.

\section{Existence and uniqueness results}

In this section, we prove existence and uniqueness of solutions for a system of HilferHadamard sequential fractional differential equations with boundary conditions (1) and (2). The following lemma concerns a linear variant of system (1) and (2).

Lemma 3.1 Let $h_{1}, h_{2} \in C([1, e], \mathbb{R})$. Then $u, v \in C([1, e], \mathbb{R})$ are solutions of the system of fractional differential equations

$$
\begin{cases}\left.{ }_{H} D_{1^{+}}^{\alpha_{1}, \beta_{1}}+k_{1 H} D_{1^{+}}^{\alpha_{1}-1, \beta_{1}}\right) u(t)=h_{1}(t), & 1<\alpha_{1} \leq 2, t \in[1, e], \\ \left({ }_{H} D_{1^{+}}^{\alpha_{2}, \beta_{2}}+k_{2 H} D_{1^{+}}^{\alpha_{2}-1, \beta_{2}}\right) v(t)=h_{2}(t), & 1<\alpha_{2} \leq 2, t \in[1, e]\end{cases}
$$

supplemented with the boundary conditions (2) if and only if

$$
\begin{aligned}
u(t)= & A_{1}(\log t)^{\gamma_{1}-1}+k_{1}\left[(\log t)^{\gamma_{1}-1} \int_{1}^{e} \frac{u(s)}{s} d s-\int_{1}^{t} \frac{u(s)}{s} d s\right] \\
& +\frac{1}{\Gamma\left(\alpha_{1}\right)}\left[\int_{1}^{t}\left(\log \frac{t}{s}\right)^{\alpha_{1}-1} \frac{h_{1}(s)}{s} d s-(\log t)^{\gamma_{1}-1} \int_{1}^{e}\left(\log \frac{e}{s}\right)^{\alpha_{1}-1} \frac{h_{1}(s)}{s} d s\right]
\end{aligned}
$$


and

$$
\begin{aligned}
v(t)= & A_{2}(\log t)^{\gamma_{2}-1}+k_{2}\left[(\log t)^{\gamma_{2}-1} \int_{1}^{e} \frac{v(s)}{s} d s-\int_{1}^{t} \frac{v(s)}{s} d s\right] \\
& +\frac{1}{\Gamma\left(\alpha_{2}\right)}\left[\int_{1}^{t}\left(\log \frac{t}{s}\right)^{\alpha_{2}-1} \frac{h_{2}(s)}{s} d s-(\log t)^{\gamma_{2}-1} \int_{1}^{e}\left(\log \frac{e}{s}\right)^{\alpha_{2}-1} \frac{h_{2}(s)}{s} d s\right] .
\end{aligned}
$$

Proof From the first equation of (5), we have

$$
{ }_{H} D_{1^{+}}^{\alpha_{1}, \beta_{1}} u(t)+k_{1 H} D_{1^{+}}^{\alpha_{1}-1, \beta_{1}} u(t)=h_{1}(t)
$$

Taking the Hadamard fractional integral of order $\alpha_{1}$ to both sides of (8), we get

$$
{ }_{H} I_{1^{+} H}^{\alpha_{1}} D_{1^{+}}^{\alpha_{1}, \beta_{1}} u(t)+k_{1 H} I_{1^{+} H}^{\alpha_{1}} D_{1^{+}}^{\alpha_{1}-1, \beta_{1}} u(t)={ }_{H} I_{1^{+}}^{\alpha_{1}} h_{1}(t)
$$

By Theorem 2.6, one has

$$
\begin{aligned}
& u(t)-\frac{\delta\left({ }_{H} I_{1^{+}}^{2-\gamma_{1}} u\right)(1)}{\Gamma\left(\gamma_{1}\right)}(\log t)^{\gamma_{1}-1}-\frac{\left({ }_{H} I_{1^{+}}^{2-\gamma_{1}} u\right)(1)}{\Gamma\left(\gamma_{1}-1\right)}(\log t)^{\gamma_{1}-2}+k_{1 H} I_{1^{+} H}^{\alpha_{1}} D_{1^{+}}^{\alpha_{1}-1, \beta_{1}} u(t) \\
& ={ }_{H} I_{1^{+}}^{\alpha_{1}} h_{1}(t) .
\end{aligned}
$$

From equation (9), by Definition 2.4, we get

$$
u(t)-\frac{\delta\left({ }_{H} I_{1^{+}}^{2-\gamma_{1}} u\right)(1)}{\Gamma\left(\gamma_{1}\right)}(\log t)^{\gamma_{1}-1}-\frac{\left({ }_{H} I_{1^{+}}^{2-\gamma_{1}} u\right)(1)}{\Gamma\left(\gamma_{1}-1\right)}(\log t)^{\gamma_{1}-2}+k_{1 H} I_{1^{+}} u(t)={ }_{H} I_{1^{+}}^{\alpha_{1}} h_{1}(t)
$$

Equation (10) can be written as follows:

$$
\begin{aligned}
u(t)= & c_{0}(\log t)^{\gamma_{1}-1}+c_{1}(\log t)^{\gamma_{1}-2} \\
& -k_{1} \int_{1}^{t} \frac{u(s)}{s} d s+\frac{1}{\Gamma\left(\alpha_{1}\right)} \int_{1}^{t}\left(\log \frac{t}{s}\right)^{\alpha_{1}-1} \frac{h_{1}(s)}{s} d s .
\end{aligned}
$$

In a similar way, one can obtain

$$
\begin{aligned}
v(t)= & d_{0}(\log t)^{\gamma_{2}-1}+d_{1}(\log t)^{\gamma_{2}-2} \\
& -k_{2} \int_{1}^{t} \frac{v(s)}{s} d s+\frac{1}{\Gamma\left(\alpha_{2}\right)} \int_{1}^{t}\left(\log \frac{t}{s}\right)^{\alpha_{2}-1} \frac{h_{2}(s)}{s} d s,
\end{aligned}
$$

where $c_{0}, c_{1}, d_{0}$, and $d_{1}$ are arbitrary constants. Now, boundary conditions (2) together with (11), (12) yield

$$
\begin{aligned}
u(1)= & c_{0}(\log 1)^{\gamma_{1}-1}+\frac{c_{1}}{(\log t)^{2-\gamma_{1}}} \\
& -k_{1} \int_{1}^{1} \frac{u(s)}{s} d s+\frac{1}{\Gamma\left(\alpha_{1}\right)} \int_{1}^{1}\left(\log \frac{1}{s}\right)^{\alpha_{1}-1} \frac{h_{1}(s)}{s} d s \\
= & 0,
\end{aligned}
$$




$$
\begin{aligned}
v(1)= & d_{0}(\log 1)^{\gamma_{2}-1}+\frac{d_{1}}{(\log t)^{2-\gamma_{2}}} \\
& -k_{2} \int_{1}^{1} \frac{v(s)}{s} d s+\frac{1}{\Gamma\left(\alpha_{2}\right)} \int_{1}^{1}\left(\log \frac{1}{s}\right)^{\alpha_{2}-1} \frac{h_{2}(s)}{s} d s
\end{aligned}
$$

$=0$,

from which we have $c_{1}=0$ and $d_{1}=0$. Equations (13) can be written as

$$
u(t)=c_{0}(\log t)^{\gamma_{1}-1}-k_{1} \int_{1}^{t} \frac{u(s)}{s} d s+\frac{1}{\Gamma\left(\alpha_{1}\right)} \int_{1}^{t}\left(\log \frac{t}{s}\right)^{\alpha_{1}-1} \frac{h_{1}(s)}{s} d s
$$

and

$$
v(t)=d_{0}(\log t)^{\gamma_{2}-1}-k_{2} \int_{1}^{t} \frac{v(s)}{s} d s+\frac{1}{\Gamma\left(\alpha_{2}\right)} \int_{1}^{t}\left(\log \frac{t}{s}\right)^{\alpha_{2}-1} \frac{h_{2}(s)}{s} d s .
$$

Next, boundary conditions (2) together with (14), (15) yield

$$
\begin{aligned}
& u(e)=c_{0}(\log e)^{\gamma_{1}-1}-k_{1} \int_{1}^{e} \frac{u(s)}{s} d s+\frac{1}{\Gamma\left(\alpha_{1}\right)} \int_{1}^{e}\left(\log \frac{e}{s}\right)^{\alpha_{1}-1} \frac{h_{1}(s)}{s} d s=A_{1}, \\
& v(e)=d_{0}(\log e)^{\gamma_{2}-1}-k_{2} \int_{1}^{e} \frac{v(s)}{s} d s+\frac{1}{\Gamma\left(\alpha_{2}\right)} \int_{1}^{e}\left(\log \frac{e}{s}\right)^{\alpha_{2}-1} \frac{h_{2}(s)}{s} d s=A_{2},
\end{aligned}
$$

from which we have

$$
\begin{aligned}
& c_{0}=A_{1}+k_{1} \int_{1}^{e} \frac{u(s)}{s} d s-\frac{1}{\Gamma\left(\alpha_{1}\right)} \int_{1}^{e}\left(\log \frac{e}{s}\right)^{\alpha_{1}-1} \frac{h_{1}(s)}{s} d s, \\
& d_{0}=A_{2}+k_{2} \int_{1}^{e} \frac{v(s)}{s} d s-\frac{1}{\Gamma\left(\alpha_{2}\right)} \int_{1}^{e}\left(\log \frac{e}{s}\right)^{\alpha_{2}-1} \frac{h_{2}(s)}{s} d s .
\end{aligned}
$$

Substituting the values of $c_{0}, c_{1}, d_{0}$, and $d_{1}$ in (11) and (12), we get integral equations (6) and (7). The converse follows by direct computation. This completes the proof.

Let us introduce the Banach space $X=C([1, e])$ endowed with the norm defined by $\|u\|:=\max _{t \in[1, e]}|u(t)|$. Thus, the product space $X \times X$ equipped with the norm $\|(u, v)\|=$ $\|u\|+\|v\|$ is a Banach space. In view of Lemma 3.1, we define an operator $\mathcal{T}: X \times X \rightarrow$ $X \times X$ by

$$
\mathcal{T}(u, v)(t)=\left(\mathcal{T}_{1}(u, v)(t), \mathcal{T}_{2}(u, v)(t)\right)
$$

where

$$
\begin{aligned}
\mathcal{T}_{1}(u, v)(t)= & A_{1}(\log t)^{\gamma_{1}-1}+k_{1}\left[(\log t)^{\gamma_{1}-1} \int_{1}^{e} \frac{u(s)}{s} d s-\int_{1}^{t} \frac{u(s)}{s} d s\right] \\
& +\frac{1}{\Gamma\left(\alpha_{1}\right)}\left[\int_{1}^{t}\left(\log \frac{t}{s}\right)^{\alpha_{1}-1} \frac{f(s, u(s), v(s))}{s} d s\right. \\
& \left.-(\log t)^{\gamma_{1}-1} \int_{1}^{e}\left(\log \frac{e}{s}\right)^{\alpha_{1}-1} \frac{f(s, u(s), v(s))}{s} d s\right]
\end{aligned}
$$


and

$$
\begin{aligned}
\mathcal{T}_{2}(u, v)(t)= & A_{2}(\log t)^{\gamma_{2}-1}+k_{2}\left[(\log t)^{\gamma_{2}-1} \int_{1}^{e} \frac{v(s)}{s} d s-\int_{1}^{t} \frac{v(s)}{s} d s\right] \\
& +\frac{1}{\Gamma\left(\alpha_{2}\right)}\left[\int_{1}^{t}\left(\log \frac{t}{s}\right)^{\alpha_{2}-1} \frac{g(s, u(s), v(s))}{s} d s\right. \\
& \left.-(\log t)^{\gamma_{2}-1} \int_{1}^{e}\left(\log \frac{e}{s}\right)^{\alpha_{2}-1} \frac{g(s, u(s), v(s))}{s} d s\right] .
\end{aligned}
$$

We need the following hypotheses in the sequel:

$\left(H_{1}\right)$ Assume that there exist real constants $m_{i}, n_{i} \geq 0(i=1,2)$ and $m_{0}>0, n_{0}>0$ such that, for all $t \in[1, e], x_{i} \in \mathbb{R}, i=1,2$,

$$
\begin{aligned}
& \left|f\left(t, x_{1}, x_{2}\right)\right| \leq m_{0}+m_{1}\left|x_{1}\right|+m_{2}\left|x_{2}\right|, \\
& \left|g\left(t, x_{1}, x_{2}\right)\right| \leq n_{0}+n_{1}\left|x_{1}\right|+n_{2}\left|x_{2}\right| .
\end{aligned}
$$

$\left(H_{2}\right)$ There exist positive constants $L, \bar{L}$, such that, for all $t \in[1, e], u_{i}, v_{i} \in \mathbb{R}, i=1,2$,

$$
\begin{gathered}
\left|f\left(t, u_{1}, u_{2}\right)-f\left(t, v_{1}, v_{2}\right)\right| \leq L\left(\left|u_{1}-v_{1}\right|+\left|u_{2}-v_{2}\right|\right), \\
\left|g\left(t, u_{1}, u_{2}\right)-g\left(t, v_{1}, v_{2}\right)\right| \leq \bar{L}\left(\left|u_{1}-v_{1}\right|+\left|u_{2}-v_{2}\right|\right) .
\end{gathered}
$$

\subsection{Existence result via Leray-Schauder alternative}

In the first theorem, we prove an existence result based on the Leray-Schauder alternative.

Theorem 3.2 Assume that $\left(H_{1}\right)$ holds. In addition it is assumed that $\max \left\{Q_{1}, Q_{2}\right\}<1$, where

$$
Q_{1}:=2\left(k_{1}+\frac{m_{1}}{\Gamma\left(\alpha_{1}+1\right)}+\frac{n_{1}}{\Gamma\left(\alpha_{2}+1\right)}\right), \quad Q_{2}:=2\left(k_{2}+\frac{m_{2}}{\Gamma\left(\alpha_{1}+1\right)}+\frac{n_{2}}{\Gamma\left(\alpha_{2}+1\right)}\right)
$$

Then system (1)-(2) has at least one solution on $[1, e]$.

Proof We will use the Leray-Schauder alternative to prove that $\mathcal{T}$, defined by (16), has a fixed point. We divide the proof into two steps.

Step I: We show that the operator $\mathcal{T}: X \times X \rightarrow X \times X$, defined by (16), is completely continuous.

First we show that $\mathcal{T}$ is continuous. Let $\left\{\left(u_{n}, v_{n}\right)\right\}$ be a sequence such that $\left(u_{n}, v_{n}\right) \rightarrow(u, v)$ in $X \times X$. Then, for each $t \in[1, e]$, we have

$$
\begin{aligned}
& \left|\mathcal{T}_{1}\left(u_{n}, v_{n}\right)(t)-\mathcal{T}_{1}(u, v)(t)\right| \\
& \leq k_{1}\left[\left|(\log t)^{\gamma_{1}-1}\right|\left|\int_{1}^{e} \frac{\left(u_{n}(s)-u(s)\right)}{s} d s\right|+\left|\int_{1}^{t} \frac{\left(u_{n}(s)-u(s)\right)}{s} d s\right|\right] \\
& \quad+\frac{1}{\Gamma\left(\alpha_{1}\right)}\left[\left|\int_{1}^{t}\left(\log \frac{t}{s}\right)^{\alpha_{1}-1} \frac{\left(f\left(s, u_{n}(s), v_{n}(s)\right)-f(s, u(s), v(s))\right)}{s} d s\right|\right. \\
& \left.\quad+\left|(\log t)^{\gamma_{1}-1}\right|\left|\int_{1}^{e}\left(\log \frac{e}{s}\right)^{\alpha_{1}-1} \frac{\left(f\left(s, u_{n}(s), v_{n}(s)\right)-f(s, u(s), v(s))\right)}{s} d s\right|\right]
\end{aligned}
$$




$$
\begin{aligned}
\leq & k_{1}\left[\int_{1}^{e} \frac{\left|u_{n}(s)-u(s)\right|}{s} d s+\int_{1}^{t} \frac{\left|u_{n}(s)-u(s)\right|}{s} d s\right] \\
& +\frac{1}{\Gamma\left(\alpha_{1}\right)}\left[\int_{1}^{t}\left(\log \frac{t}{s}\right)^{\alpha_{1}-1} \frac{\left|f\left(s, u_{n}(s), v_{n}(s)\right)-f(s, u(s), v(s))\right|}{s} d s\right. \\
& \left.+\int_{1}^{e}\left(\log \frac{e}{s}\right)^{\alpha_{1}-1} \frac{\left|f\left(s, u_{n}(s), v_{n}(s)\right)-f(s, u(s), v(s))\right|}{s} d s\right]
\end{aligned}
$$

Since $f$ is continuous, we get

$$
\left|f\left(s, u_{n}(s), v_{n}(s)\right)-f(s, u(s), v(s))\right| \rightarrow 0 \quad \text { as }\left(u_{n}, v_{n}\right) \rightarrow(u, v) .
$$

Then

$$
\left\|\mathcal{T}_{1}\left(u_{n}, v_{n}\right)-\mathcal{T}_{1}(u, v)\right\| \rightarrow 0 \quad \text { as }\left(u_{n}, v_{n}\right) \rightarrow(u, v)
$$

In the same way, we obtain

$$
\left\|\mathcal{T}_{2}\left(u_{n}, v_{n}\right)-\mathcal{T}_{2}(u, v)\right\| \rightarrow 0 \quad \text { as }\left(u_{n}, v_{n}\right) \rightarrow(u, v)
$$

It follows from (19) and (20) that $\left\|\mathcal{T}\left(u_{n}, v_{n}\right)-\mathcal{T}(u, v)\right\| \rightarrow 0$ as $\left(u_{n}, v_{n}\right) \rightarrow(u, v)$. Hence $\mathcal{T}$ is continuous.

Now we show that $\mathcal{T}$ is compact. Let $\Omega \subset X \times X$ be bounded. Then there exist positive constants $L_{1}$ and $L_{2}$ such that $|f(t, u(t), v(t))| \leq L_{1},|g(t, u(t), v(t))| \leq L_{2}, \forall(u, v) \in \Omega$. Let $(u, v) \in \Omega$. Then there exists $M$ such that $\|(u, v)\|=\|u\|+\|v\| \leq M, \forall(u, v) \in \Omega$. We have

$$
\begin{aligned}
& \left|\mathcal{T}_{1}(u, v)(t)\right| \\
& \leq A_{1}+k_{1}\left[\int_{1}^{e} \frac{|u(s)|}{s} d s+\int_{1}^{t} \frac{|u(s)|}{s} d s\right] \\
& \quad+\frac{1}{\Gamma\left(\alpha_{1}\right)}\left[\int_{1}^{t}\left(\log \frac{t}{s}\right)^{\alpha_{1}-1} \frac{|f(s, u(s), v(s))|}{s} d s+\int_{1}^{e}\left(\log \frac{e}{s}\right)^{\alpha_{1}-1} \frac{|f(s, u(s), v(s))|}{s} d s\right] \\
& \leq A_{1}+k_{1}\left[\int_{1}^{e} \frac{\max _{s \in[1, e]}|u(s)|}{s} d s+\int_{1}^{t} \frac{\max _{s \in[1, e]}|u(s)|}{s} d s\right] \\
& \quad+\frac{L_{1}}{\Gamma\left(\alpha_{1}\right)}\left[\int_{1}^{t}\left(\log \frac{t}{s}\right)^{\alpha_{1}-1} \frac{d s}{s}+\int_{1}^{e}\left(\log \frac{e}{s}\right)^{\alpha_{1}-1} \frac{d s}{s}\right] \\
& \leq A_{1}+k_{1}\|u\|[1+(\log e)]+\frac{L_{1}}{\Gamma\left(\alpha_{1}+1\right)}\left[(\log e)^{\alpha_{1}}+1\right]
\end{aligned}
$$

which, on taking the norm for $t \in[1, e]$, yields

$$
\left\|\mathcal{T}_{1}\left(u_{n}, v_{n}\right)\right\| \leq A_{1}+2\left[k_{1}\|u\|+\frac{L_{1}}{\Gamma\left(\alpha_{1}+1\right)}\right]
$$

In the same way, we obtain

$$
\left\|\mathcal{T}_{2}\left(u_{n}, v_{n}\right)\right\| \leq A_{2}+2\left[k_{2}\|v\|+\frac{L_{2}}{\Gamma\left(\alpha_{2}+1\right)}\right]
$$


It follows that

$$
\begin{aligned}
\|\mathcal{T}(u, v)\| & \leq A_{1}+A_{2}+2\left[k_{1}\|u\|+k_{2}\|v\|+\frac{L_{1}}{\Gamma\left(\alpha_{1}+1\right)}+\frac{L_{2}}{\Gamma\left(\alpha_{2}+1\right)}\right] \\
& \leq A_{1}+A_{2}+2\left[M\left(k_{1}+k_{2}\right)+\frac{L_{1}}{\Gamma\left(\alpha_{1}+1\right)}+\frac{L_{2}}{\Gamma\left(\alpha_{2}+1\right)}\right] .
\end{aligned}
$$

This mean that there is $P=A_{1}+A_{2}+2\left[M\left(k_{1}+k_{2}\right)+\frac{L_{1}}{\Gamma\left(\alpha_{1}+1\right)}+\frac{L_{2}}{\Gamma\left(\alpha_{2}+1\right)}\right]$ such that $\|\mathcal{T}(u, v)\| \leq P$. Hence $\mathcal{T}$ is uniformly bounded.

Finally we show that $\mathcal{T}$ is equicontinuous. Let $t, t_{0} \in[1, e]$ with $t_{0}<t$. Then we have

$$
\begin{aligned}
& \left|\mathcal{T}_{1}(u, v)(t)-\mathcal{T}_{1}(u, v)\left(t_{0}\right)\right| \\
& \leq A_{1}\left[(\log t)^{\gamma_{1}-1}-\left(\log t_{0}\right)^{\gamma_{1}-1}\right] \\
& +k_{1}\left[\left((\log t)^{\gamma_{1}-1}-\left(\log t_{0}\right)^{\gamma_{1}-1}\right) \int_{1}^{e} \frac{|u(s)|}{s} d s+\int_{t_{0}}^{t} \frac{|u(s)|}{s} d s\right] \\
& +\frac{1}{\Gamma\left(\alpha_{1}\right)}\left[\int_{1}^{t_{0}}\left(\left(\log \frac{t}{s}\right)^{\alpha_{1}-1}-\left(\log \frac{t_{0}}{s}\right)^{\alpha_{1}-1}\right) \frac{|f(s, u(s), v(s))|}{s} d s\right. \\
& +\int_{t_{0}}^{t}\left(\log \frac{t}{s}\right)^{\alpha_{1}-1} \frac{|f(s, u(s), v(s))|}{s} d s \\
& \left.+\left((\log t)^{\gamma_{1}-1}-\left(\log t_{0}\right)^{\gamma_{1}-1}\right) \int_{1}^{e}\left(\log \frac{e}{s}\right)^{\alpha_{1}-1} \frac{|f(s, u(s), v(s))|}{s} d s\right] \\
& \leq A_{1}\left[(\log t)^{\gamma_{1}-1}-\left(\log t_{0}\right)^{\gamma_{1}-1}\right] \\
& +k_{1}\left[\|u\|\left((\log t)^{\gamma_{1}-1}-\left(\log t_{0}\right)^{\gamma_{1}-1}\right)+\|u\|\left(\log t-\log t_{0}\right)\right] \\
& +\frac{L_{1}}{\Gamma\left(\alpha_{1}\right)}\left[\int_{1}^{t_{0}}\left(\log \frac{t}{s}\right)^{\alpha_{1}-1} \frac{d s}{s}-\int_{1}^{t_{0}}\left(\log \frac{t_{0}}{s}\right)^{\alpha_{1}-1} \frac{d s}{s}+\int_{t_{0}}^{t}\left(\log \frac{t}{s}\right)^{\alpha_{1}-1} \frac{d s}{s}\right. \\
& \left.+\left((\log t)^{\gamma_{1}-1}-\left(\log t_{0}\right)^{\gamma_{1}-1}\right) \int_{1}^{e}\left(\log \frac{e}{s}\right)^{\alpha_{1}-1} \frac{d s}{s}\right] \\
& \leq A_{1}\left[(\log t)^{\gamma_{1}-1}-\left(\log t_{0}\right)^{\gamma_{1}-1}\right] \\
& +k_{1} M\left[\left((\log t)^{\gamma_{1}-1}-\left(\log t_{0}\right)^{\gamma_{1}-1}\right)+\left(\log t-\log t_{0}\right)\right] \\
& +\frac{L_{1}}{\Gamma\left(\alpha_{1}+1\right)}\left[\left((\log t)^{\gamma_{1}-1}-\left(\log t_{0}\right)^{\gamma_{1}-1}\right)+\left((\log t)^{\alpha_{1}}-\left(\log t_{0}\right)^{\alpha_{1}}\right)\right]
\end{aligned}
$$

and

$$
\begin{aligned}
& \left|\mathcal{T}_{2}(u, v)(t)-\mathcal{T}_{2}(u, v)\left(t_{0}\right)\right| \\
& \leq A_{2}\left[(\log t)^{\gamma_{2}-1}-\left(\log t_{0}\right)^{\gamma_{2}-1}\right] \\
& \quad+k_{2}\left[\left((\log t)^{\gamma_{2}-1}-\left(\log t_{0}\right)^{\gamma_{2}-1}\right) \int_{1}^{e} \frac{|v(s)|}{s} d s+\int_{t_{0}}^{t} \frac{|v(s)|}{s} d s\right] \\
& \quad+\frac{1}{\Gamma\left(\alpha_{2}\right)}\left[\int_{1}^{t_{0}}\left(\left(\log \frac{t}{s}\right)^{\alpha_{2}-1}-\left(\log \frac{t_{0}}{s}\right)^{\alpha_{2}-1}\right) \frac{|g(s, u(s), v(s))|}{s} d s\right. \\
& \quad+\int_{t_{0}}^{t}\left(\log \frac{t}{s}\right)^{\alpha_{2}-1} \frac{|g(s, u(s), v(s))|}{s} d s
\end{aligned}
$$




$$
\begin{aligned}
& \left.+\left((\log t)^{\gamma_{2}-1}-\left(\log t_{0}\right)^{\gamma_{2}-1}\right) \int_{1}^{e}\left(\log \frac{e}{s}\right)^{\alpha_{2}-1} \frac{|g(s, u(s), v(s))|}{s} d s\right] \\
\leq & A_{2}\left[(\log t)^{\gamma_{2}-1}-\left(\log t_{0}\right)^{\gamma_{2}-1}\right] \\
& +k_{2}\left[\|v\|\left((\log t)^{\gamma_{2}-1}-\left(\log t_{0}\right)^{\gamma_{2}-1}\right)+\|v\|\left(\log t-\log t_{0}\right)\right] \\
& +\frac{L_{2}}{\Gamma\left(\alpha_{2}\right)}\left[\int_{1}^{t_{0}}\left(\log \frac{t}{s}\right)^{\alpha_{2}-1} \frac{d s}{s}-\int_{1}^{t_{0}}\left(\log \frac{t_{0}}{s}\right)^{\alpha_{2}-1} \frac{d s}{s}+\int_{t_{0}}^{t}\left(\log \frac{t}{s}\right)^{\alpha_{2}-1} \frac{d s}{s}\right. \\
& \left.+\left((\log t)^{\gamma_{2}-1}-\left(\log t_{0}\right)^{\gamma_{2}-1}\right) \int_{1}^{e}\left(\log \frac{e}{s}\right)^{\alpha_{2}-1} \frac{d s}{s}\right] \\
\leq & A_{2}\left[(\log t)^{\gamma_{2}-1}-\left(\log t_{0}\right)^{\gamma_{2}-1}\right] \\
& +k_{2} M\left[\left((\log t)^{\gamma_{2}-1}-\left(\log t_{0}\right)^{\gamma_{2}-1}\right)+\left(\log t-\log t_{0}\right)\right] \\
& +\frac{L_{2}}{\Gamma\left(\alpha_{2}+1\right)}\left[\left((\log t)^{\gamma_{2}-1}-\left(\log t_{0}\right)^{\gamma_{2}-1}\right)+\left((\log t)^{\alpha_{2}}-\left(\log t_{0}\right)^{\alpha_{2}}\right)\right] .
\end{aligned}
$$

Take $t \rightarrow t_{0}$, from (21) and (22), we have

$$
\left|\mathcal{T}_{1}(u, v)(t)-\mathcal{T}_{1}(u, v)\left(t_{0}\right)\right| \rightarrow 0 \quad \text { and } \quad\left|\mathcal{T}_{2}(u, v)(t)-\mathcal{T}_{2}(u, v)\left(t_{0}\right)\right| \rightarrow 0 \quad \text { as } t \rightarrow t_{0}
$$

Hence $\mathcal{T}$ is equicontinuous. By Arzelá-Ascoli theorem, we get that $\mathcal{T}(\Omega)$ is compact, that is, $\mathcal{T}$ is compact on $\Omega$. Therefore $\mathcal{T}$ is completely continuous.

Step II: We show that the set $\varepsilon=\{(u, v) \in X \times X \mid(u, v)=\lambda \mathcal{T}(u, v), 0 \leq \lambda \leq 1\}$ is bounded. Let $(u, v) \in \varepsilon$, then $(u, v)=\lambda \mathcal{T}(u, v)$. For any $t \in[1, e]$, we have $u(t)=\lambda \mathcal{T}_{1}(u, v)(t), v(t)=$ $\lambda \mathcal{T}_{2}(u, v)(t)$. Then, in view of assumption $\left(H_{1}\right)$, we obtain

$$
\begin{aligned}
|u(t)| \leq & \left|\mathcal{T}_{1}(u, v)(t)\right| \\
\leq & A_{1}+k_{1}\left[\int_{1}^{e} \frac{|u(s)|}{s} d s+\int_{1}^{t} \frac{|u(s)|}{s} d s\right] \\
& +\frac{1}{\Gamma\left(\alpha_{1}\right)}\left[\int_{1}^{t}\left(\log \frac{t}{s}\right)^{\alpha_{1}-1} \frac{|f(s, u(s), v(s))|}{s} d s\right. \\
& \left.+\int_{1}^{e}\left(\log \frac{e}{s}\right)^{\alpha_{1}-1} \frac{f(s, u(s), v(s)) \mid}{s} d s\right] \\
\leq & A_{1}+k_{1}\left[\|u\| \int_{1}^{e} \frac{d s}{s}+\|u\| \int_{1}^{t} \frac{d s}{s}\right] \\
& +\frac{\left(m_{0}+m_{1}\|u\|+m_{2}\|v\|\right)}{\Gamma\left(\alpha_{1}\right)}\left[\int_{1}^{t}\left(\log \frac{t}{s}\right)^{\alpha_{1}-1} \frac{d s}{s}+\int_{1}^{e}\left(\log \frac{e}{s}\right)^{\alpha_{1}-1} \frac{d s}{s}\right] \\
\leq & A_{1}+k_{1}\|u\|[1+(\log e)]+\frac{\left(m_{0}+m_{1}\|u\|+m_{2}\|v\|\right)}{\Gamma\left(\alpha_{1}+1\right)}\left[(\log e)^{\alpha_{1}}+1\right],
\end{aligned}
$$

which, on taking maximum for $t \in[1, e]$, yields

$$
\|u\| \leq A_{1}+2 k_{1}\|u\|+2\left(\frac{m_{0}+m_{1}\|u\|+m_{2}\|v\|}{\Gamma\left(\alpha_{1}+1\right)}\right) .
$$


In a similar manner, one can obtain

$$
\|v\| \leq A_{2}+2 k_{2}\|v\|+2\left(\frac{n_{0}+n_{1}\|u\|+n_{2}\|v\|}{\Gamma\left(\alpha_{2}+1\right)}\right) .
$$

From (23) and (24), we have

$$
\begin{aligned}
\|(u, v)\|= & \|u\|+\|v\| \\
\leq & A_{1}+A_{2}+\frac{2 m_{0}}{\Gamma\left(\alpha_{1}+1\right)}+\frac{2 n_{0}}{\Gamma\left(\alpha_{2}+1\right)} \\
& +2\left(k_{1}+\frac{m_{1}}{\Gamma\left(\alpha_{1}+1\right)}+\frac{n_{1}}{\Gamma\left(\alpha_{2}+1\right)}\right)\|u\| \\
& +2\left(k_{2}+\frac{m_{2}}{\Gamma\left(\alpha_{1}+1\right)}+\frac{n_{2}}{\Gamma\left(\alpha_{2}+1\right)}\right)\|v\| \\
\leq & A_{1}+A_{2}+\frac{2 m_{0}}{\Gamma\left(\alpha_{1}+1\right)}+\frac{2 n_{0}}{\Gamma\left(\alpha_{2}+1\right)}+\max \left\{Q_{1}, Q_{2}\right\}\|(u, v)\|,
\end{aligned}
$$

and consequently,

$$
\|(u, v)\| \leq \frac{A_{1}+A_{2}+\frac{2 m_{0}}{\Gamma\left(\alpha_{1}+1\right)}+\frac{2 n_{0}}{\Gamma\left(\alpha_{2}+1\right)}}{1-\max \left\{Q_{1}, Q_{2}\right\}} .
$$

Therefore the set $\varepsilon$ is bounded. By Theorem 2.7, we get that the operator $\mathcal{T}$ has at least one fixed point. Therefore, problem (1)-(2) has at least one solution on $[1, e]$.

\subsection{Existence and uniqueness result via the Banach fixed point theorem}

Next, we prove an existence and uniqueness result based on the Banach contraction mapping principle.

Theorem 3.3 Assume that $\left(H_{2}\right)$ holds. Then system (1)-(2) has a unique solution on [1,e] provided that

$$
\mu:=2\left(k_{1}+k_{2}+\frac{L}{\Gamma\left(\alpha_{1}+1\right)}+\frac{\bar{L}}{\Gamma\left(\alpha_{2}+1\right)}\right)<1 .
$$

Proof We will use the Banach fixed point theorem to prove that $\mathcal{T}$, defined by (16), has a unique fixed point. Fixing $N_{1}=\max _{t \in[1, e]}|f(t, 0,0)|<\infty, N_{2}=\max _{t \in[1, e]}|g(t, 0,0)|<\infty$ and using assumption $\left(H_{2}\right)$, we obtain

$$
\begin{aligned}
& |f(t, u(t), v(t))|=|f(t, u(t), v(t))-f(t, 0,0)+f(t, 0,0)| \leq L(\|u\|+\|v\|)+N_{1}, \\
& |g(t, u(t), v(t))|=|g(t, u(t), v(t))-g(t, 0,0)+g(t, 0,0)| \leq \bar{L}(\|u\|+\|v\|)+N_{2} .
\end{aligned}
$$

We choose

$$
r \geq \frac{A_{1}+A_{2}+2\left(\frac{N_{1}}{\Gamma\left(\alpha_{1}+1\right)}+\frac{N_{2}}{\Gamma\left(\alpha_{2}+1\right)}\right)}{1-2\left(k_{1}+k_{2}+\frac{L}{\Gamma\left(\alpha_{1}+1\right)}+\frac{\bar{L}}{\Gamma\left(\alpha_{2}+1\right)}\right)} .
$$

We divide the proof into two steps.

Step $I$ : First we show that $\mathcal{T}\left(B_{r}\right) \subset B_{r}$, where $B_{r}=\{(u, v) \in X \times X:\|(u, v)\| \leq r\}$. 
Let $(u, v) \in B_{r}$. Then, using (26), we obtain

$$
\begin{aligned}
\left|\mathcal{T}_{1}(u, v)(t)\right| \leq & A_{1}+k_{1}\left[\int_{1}^{e} \frac{|u(s)|}{s} d s+\int_{1}^{t} \frac{|u(s)|}{s} d s\right] \\
& +\frac{1}{\Gamma\left(\alpha_{1}\right)}\left[\int_{1}^{t}\left(\log \frac{t}{s}\right)^{\alpha_{1}-1} \frac{|f(s, u(s), v(s))|}{s} d s\right. \\
& \left.+\int_{1}^{e}\left(\log \frac{e}{s}\right)^{\alpha_{1}-1} \frac{|f(s, u(s), v(s))|}{s} d s\right] \\
\leq & A_{1}+k_{1}\left[\int_{1}^{e} \frac{\max _{s \in[1, e]}|u(s)|}{s} d s+\int_{1}^{t} \frac{\max _{s \in[1, e]}|u(s)|}{s} d s\right] \\
& +\frac{L(\|u\|+\|v\|)+N_{1}}{\Gamma\left(\alpha_{1}\right)}\left[\int_{1}^{t}\left(\log \frac{t}{s}\right)^{\alpha_{1}-1} \frac{d s}{s}+\int_{1}^{e}\left(\log \frac{e}{s}\right)^{\alpha_{1}-1} \frac{d s}{s}\right] \\
\leq & A_{1}+2 k_{1} r+\frac{2}{\Gamma\left(\alpha_{1}+1\right)}\left(L r+N_{1}\right),
\end{aligned}
$$

which, on taking the norm for $t \in[1, e]$, yields

$$
\left\|\mathcal{T}_{1}(u, v)\right\| \leq A_{1}+2 k_{1} r+\frac{2}{\Gamma\left(\alpha_{1}+1\right)}\left(L r+N_{1}\right)
$$

In the same way, one has

$$
\left\|\mathcal{T}_{2}(u, v)\right\| \leq A_{2}+2 k_{2} r+\frac{2}{\Gamma\left(\alpha_{2}+1\right)}\left(\bar{L} r+N_{2}\right) .
$$

Then we have

$$
\begin{aligned}
\|\mathcal{T}(u, v)\| \leq & A_{1}+A_{2}+2\left(k_{1}+k_{2}\right) r \\
& +2\left(\frac{L}{\Gamma\left(\alpha_{1}+1\right)}+\frac{\bar{L}}{\Gamma\left(\alpha_{2}+1\right)}\right) r+2\left(\frac{N_{1}}{\Gamma\left(\alpha_{1}+1\right)}+\frac{N_{2}}{\Gamma\left(\alpha_{2}+1\right)}\right) \\
\leq & r .
\end{aligned}
$$

Thus $\|\mathcal{T}(u, v)\| \leq r$, that is, $\mathcal{T}(u, v) \in B_{r}$. Hence $\mathcal{T}\left(B_{r}\right) \subset B_{r}$.

Step II : We show that the operator $\mathcal{T}$ is a contraction.

Let $\left(u_{2}, v_{2}\right),\left(u_{1}, v_{1}\right) \in X \times X$. Then, for any $t \in[1, e]$, we have

$$
\begin{aligned}
& \left|\mathcal{T}_{1}\left(u_{2}, v_{2}\right)(t)-\mathcal{T}_{1}\left(u_{1}, v_{1}\right)(t)\right| \\
& \leq k_{1}\left[\int_{1}^{e} \frac{\left|u_{2}(s)-u_{1}(s)\right|}{s} d s+\int_{1}^{t} \frac{\left|u_{2}(s)-u_{1}(s)\right|}{s} d s\right] \\
& \quad+\frac{1}{\Gamma\left(\alpha_{1}\right)}\left[\int_{1}^{t}\left(\log \frac{t}{s}\right)^{\alpha_{1}-1} \frac{\left|f\left(s, u_{2}(s), v_{2}(s)\right)-f\left(s, u_{1}(s), v_{1}(s)\right)\right|}{s} d s\right. \\
& \left.\quad+\int_{1}^{e}\left(\log \frac{e}{s}\right)^{\alpha_{1}-1} \frac{\left|f\left(s, u_{2}(s), v_{2}(s)\right)-f\left(s, u_{1}(s), v_{1}(s)\right)\right|}{s} d s\right] \\
& \leq 2 k_{1}\left\|u_{2}-u_{1}\right\|+\frac{2 L}{\Gamma\left(\alpha_{1}+1\right)}\left(\left\|u_{2}-u_{1}\right\|+\left\|v_{2}-v_{1}\right\|\right) \\
& \leq 2 k_{1}\left(\left\|u_{2}-u_{1}\right\|+\left\|v_{2}-v_{1}\right\|\right)+\frac{2 L}{\Gamma\left(\alpha_{1}+1\right)}\left(\left\|u_{2}-u_{1}\right\|+\left\|v_{2}-v_{1}\right\|\right)
\end{aligned}
$$


which, on taking the norm for $t \in[1, e]$, yields

$$
\left\|\mathcal{T}_{1}\left(u_{2}, v_{2}\right)-\mathcal{T}_{1}\left(u_{1}, v_{1}\right)\right\| \leq\left(2 k_{1}+\frac{2 L}{\Gamma\left(\alpha_{1}+1\right)}\right)\left(\left\|u_{2}-u_{1}\right\|+\left\|v_{2}-v_{1}\right\|\right) .
$$

Similarly,

$$
\left\|\mathcal{T}_{2}\left(u_{2}, v_{2}\right)-\mathcal{T}_{2}\left(u_{1}, v_{1}\right)\right\| \leq\left(2 k_{2}+\frac{2 \bar{L}}{\Gamma\left(\alpha_{1}+1\right)}\right)\left(\left\|u_{2}-u_{1}\right\|+\left\|v_{2}-v_{1}\right\|\right) .
$$

It follows from (27) and (28) that $\left\|\mathcal{T}\left(u_{2}, v_{2}\right)-\mathcal{T}\left(u_{1}, v_{1}\right)\right\| \leq \mu\left(\left\|u_{2}-u_{1}\right\|+\left\|v_{2}-v_{1}\right\|\right)$, which, in view of (25), shows that the operator $\mathcal{T}$ is a contraction. From Steps I and II, by Theorem 2.8, we get that the operator $\mathcal{T}$ has a unique fixed point. Therefore system (1)-(2) has a unique solution on $[1, e]$.

\section{Examples}

In this section, we give two examples to illustrate our main results.

Example 4.1 Consider the following system:

$$
\begin{cases}\left({ }_{H} D^{\frac{3}{2}, \frac{1}{2}}+\frac{1}{6}{ }_{H} D^{\frac{1}{2}, \frac{1}{2}}\right) u(t)=\frac{|u(t)|}{(t+3)^{4}(1+|u(t)|)}+\frac{|v(t)|}{90(1+|v(t)|)}+\frac{1}{16}, & t \in[1, e], \\ \left({ }_{H} D^{\frac{3}{2}, \frac{1}{2}}+\frac{1}{8}{ }_{H} D^{\frac{1}{2}, \frac{1}{2}}\right) v(t)=\frac{\sin (\pi(t))}{80 \pi}+\frac{1}{15 \sqrt{t+8}}+\frac{|v(t)|}{100(1+|v(t)|)}, & t \in[1, e], \\ u(1)=0, \quad u(e)=\frac{1}{2}, \quad v(1)=0, \quad v(e)=\frac{1}{4} . & \end{cases}
$$

Here $\alpha_{1}=\frac{3}{2}, \alpha_{2}=\frac{3}{2}, \beta_{1}=\frac{1}{2}, \beta_{2}=\frac{1}{2}, A_{1}=\frac{1}{2}, A_{2}=\frac{1}{4}, k_{1}=\frac{1}{6}, k_{2}=\frac{1}{8}$.

We see that $\left(H_{1}\right)$ holds, because

$$
|f(t, u, v)| \leq \frac{1}{16}+\frac{1}{256}|u|+\frac{1}{90}|v| \quad \text { and } \quad|g(t, u, v)| \leq \frac{1}{45}+\frac{1}{80}|u|+\frac{1}{100}|v|,
$$

with

$$
m_{0}=\frac{1}{16}, \quad m_{1}=\frac{1}{256}, \quad m_{2}=\frac{1}{90}, \quad n_{0}=\frac{1}{45}, \quad n_{1}=\frac{1}{80}, \quad n_{2}=\frac{1}{100} .
$$

In addition, $Q_{1} \approx 0.3580<1, Q_{2} \approx 0.2818<1$, and $\max \left\{Q_{1}, Q_{2}\right\} \approx 0.6420$. Thus, the hypotheses of Theorem 3.2 are satisfied. Therefore, by Theorem 3.2, system (29) has at least one solution on $[1, e]$.

Example 4.2 Consider the following Hilfer-Hadamard system:

$$
\left\{\begin{array}{l}
\left({ }_{H} D^{\frac{5}{4}, \frac{1}{2}}+\frac{1}{7} H_{H} D^{\frac{1}{4}, \frac{1}{2}}\right) u(t)=(1+\log t)\left(\frac{|u(t)|}{100+|u(t)|}\right)+\frac{|v(t)|}{(8+t)^{3}(1+|v(t)|)}+\frac{1}{\sqrt{t+15}}, \quad t \in[1, e], \\
\left({ }_{H} D^{\frac{3}{2}, \frac{1}{2}}+\frac{1}{9}{ }_{H} D^{\frac{1}{2}, \frac{1}{2}}\right) v(t)=\frac{\sin (u(t))}{(7+t)^{3}}+\frac{7}{49+t^{2}}+\frac{|v(t)|}{\sqrt{99+t^{2}}(4+|v(t)|)}, \quad t \in[1, e], \\
u(1)=0, \quad u(e)=\frac{1}{3}, \quad v(1)=0, \quad v(e)=\frac{1}{5} .
\end{array}\right.
$$

Here $\alpha_{1}=\frac{5}{4}, \alpha_{2}=\frac{3}{2}, \beta_{1}=\frac{1}{2}, \beta_{2}=\frac{1}{2}, A_{1}=\frac{1}{3}, A_{2}=\frac{1}{5}, k_{1}=\frac{1}{7}, k_{2}=\frac{1}{9}$.

Note that $\left(H_{2}\right)$ holds, because

$$
\left|f\left(t, u_{1}, u_{2}\right)-f\left(t, v_{1}, v_{2}\right)\right| \leq \frac{1}{50}\left(\left|u_{1}-v_{1}\right|+\left|u_{2}-v_{2}\right|\right)
$$


and

$$
\left|g\left(t, u_{1}, u_{2}\right)-g\left(t, v_{1}, v_{2}\right)\right| \leq \frac{1}{40}\left(\left|u_{1}-v_{1}\right|+\left|u_{2}-v_{2}\right|\right),
$$

with $L=\frac{1}{50}, \bar{L}=\frac{1}{40}$. In addition,

$$
\mu:=2\left(k_{1}+k_{2}+\frac{L}{\Gamma\left(\alpha_{1}+1\right)}+\frac{\bar{L}}{\Gamma\left(\alpha_{2}+1\right)}\right) \approx 0.580854<1 .
$$

Thus, all the conditions of Theorem 3.3 are satisfied. Therefore, by Theorem 3.3, system (30) has a unique solution on $[1, e]$.

\section{Conclusion}

In this paper, we studied existence and uniqueness of solutions for a system of HilferHadamard sequential fractional differential equations with two-point boundary conditions. The existence result is proved by using the Leray-Schauder alternative while the Banach contraction mapping principle is used to obtain the existence and uniqueness result. Examples illustrating the obtained results are also presented. Our results on a system of Hilfer-Hadamard fractional derivatives are new in the given configuration. We emphasize that we used Hilfer-Hadamard derivative of order $1<\alpha_{i} \leq 2, i=1,2$. In the context of sequential fractional differential equations with two-point boundary conditions, the present paper significantly contributes to the existing literature on the topic. The problems studied in this paper can be extended to cover other kinds of boundary conditions.

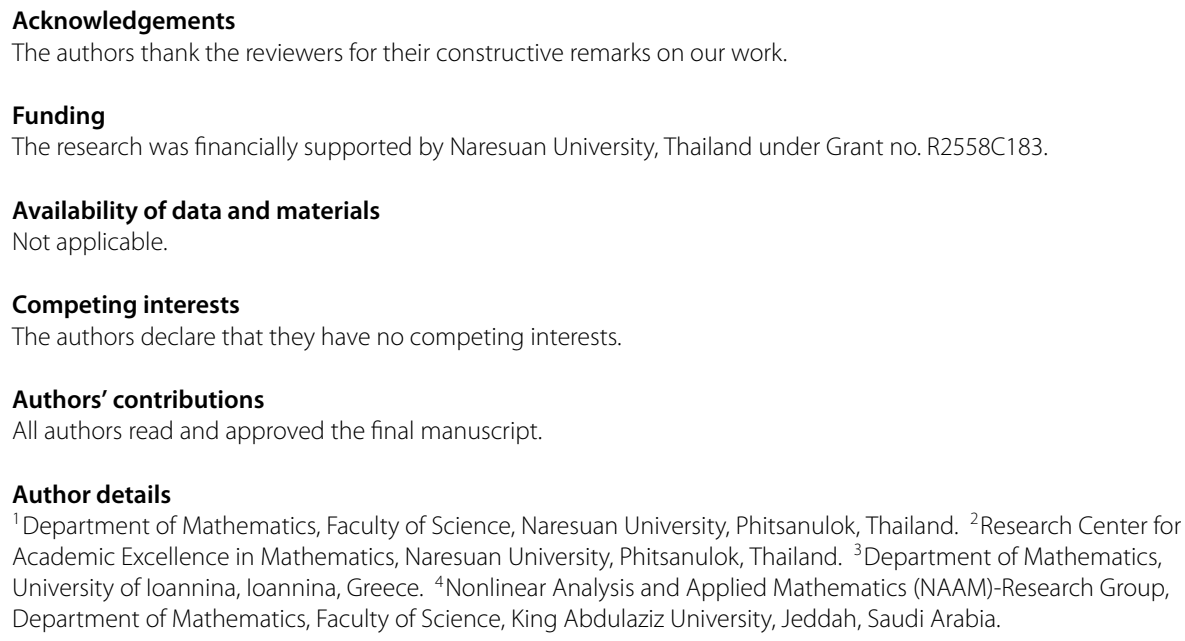

\section{Publisher's Note}

Springer Nature remains neutral with regard to jurisdictional claims in published maps and institutional affiliations.

Received: 12 August 2019 Accepted: 10 December 2019 Published online: 18 December 2019

\section{References}

1. Kilbas, A.A., Srivastava, H.M., Trujillo, J.J.: Theory and Applications of Fractional Differential Equations. Elsevier, Amsterdam (2006)

2. Podlubny, I.: Fractional Differential Equations. Academic Press, San Diego (1999)

3. Samko, S.G., Kilbas, A.A., Marichev, O.I.: Fractional Integrals and Derivatives: Theory and Applications. Gordon \& Breach, New York (1993) 
4. Miller, K.S., Ross, B.: An Introduction to the Fractional Calculus and Fractional Differential Equations. Wiley, New York (1993)

5. Diethelm, K.: The Analysis of Fractional Differential Equations. Springer, Berlin (2010)

6. Hilfer, R.: Applications of Fractional Calculus in Physics. World Scientific, Singapore (2000)

7. Fernandez, A., Baleanu, D.: On a new definition of fractional differintegrals with Mittag-Leffler kernel. Filomat 33, 245-254 (2019)

8. Baleanu, D., Jajarmi, A., Sajjadi, S.S., Mozyrska, D.: A new fractional model and optimal control of a tumor-immune surveillance with non-singular derivative operator. Chaos 29, 083127 (2019)

9. Alseadi, A., Baleanu, D., Etemad, S., Rezapour, Sh.: On coupled systems of time-fractional differential problems by using a new fractional derivative. J. Funct. Spaces 2016, Article ID 4626940 (2016)

10. Baleanu, D., Fernandez, A.: On fractional operators and their classifications. Mathematics 7(9), Article ID 830 (2019)

11. Fernandez, A., Özarslan, M.A., Baleanu, D.: On fractional calculus with general analytic kernels. Appl. Math. Comput. 354, 248-265 (2019)

12. Ge, Z.M., Ou, C.Y.: Chaos synchronization of fractional order modified Duffing systems with parameters excited by a chaotic signal. Chaos Solitons Fractals 35, 705-717 (2008)

13. Sokolov, I.M., Klafter, J., Blumen, A.: Fractional kinetics. Phys. Today 55, 48-54 (2002)

14. Javidi, M., Ahmad, B.: Dynamic analysis of time fractional order phytoplankton-toxic phytoplankton-zooplankton system. Ecol. Model. 318, 8-18 (2015)

15. Petras, I., Magin, R.L.: Simulation of drug uptake in a two compartmental fractional model for a biological system. Commun. Nonlinear Sci. Numer. Simul. 16, 4588-4595 (2011)

16. Ahmad, B., Nieto, J.J.: Existence results for a coupled system of nonlinear fractional differential equations with three-point boundary conditions. Comput. Math. Appl. 58, 1838-1843 (2009)

17. Goodrich, C.S.: Existence of a positive solution to systems of differential equations of fractional order. Comput. Math Appl. 62, 1251-1268 (2011)

18. Ntouyas, S.K., Obaid, M.: A coupled system of fractional differential equations with nonlocal integral boundary conditions. Adv. Differ. Equ. 2012, 130 (2012)

19. Su, X.: Boundary value problem for a coupled system of nonlinear fractional differential equations. Appl. Math. Lett. 22, 64-69 (2009)

20. Ahmad, B., Ntouyas, S.K.: Existence results for a coupled system of Caputo type sequential fractional differential equations with nonlocal integral boundary conditions. Appl. Math. Comput. 266, 615-622 (2015)

21. Ahmad, B., Ntouyas, S.K.: Existence results for Caputo type sequential fractional differential inclusions with nonlocal integral boundary conditions. J. Appl. Math. Comput. 50, 157-174 (2016)

22. Ahmad, B., Ntouyas, S.K., Alsaedi, A.: On a coupled system of fractional differential equations with coupled nonlocal and integral boundary conditions. Chaos Solitons Fractals 83, 234-241 (2016)

23. Alsaedi, A., Aljoudi, S., Ahmad, B.: Existence of solutions for Riemann-Liouville type coupled systems of fractional integro-differential equations and boundary conditions. Electron. J. Differ. Equ. 2016, Article ID 211 (2016)

24. Alsulami, H.H., Ntouyas, S.K., Agarwal, R.P., Ahmad, B., Alsaedi, A.: A study of fractional-order coupled systems with a new concept of coupled non-separated boundary conditions. Bound. Value Probl. 2017, 68 (2017)

25. Ahmad, B., Nieto, J.J., Alsaedi, A., Aqlan, M.H.: A coupled system of Caputo-type sequential fractional differential equations with coupled (periodic/anti-periodic type) boundary conditions. Mediterr. J. Math. 14, Article ID 227 (2017). https://doi.org/10.1007/s00009-017-1027-2

26. Shiri, B., Baleanu, D.: System of fractional differential algebraic equations with applications. Chaos Solitons Fractals 120, 203-212 (2019)

27. Baleanu, D., Shiri, B., Srivastava, H.M., Al Qurashi, M.: A Chebyshev spectral method based on operational matrix for fractional differential equations involving non-singular Mittag-Leffler kernel. Adv. Differ. Equ. 2018, 353 (2018)

28. Baleanu, D., Shiri, B.: Collocation methods for fractional differential equations involving non-singular kernel. Chaos Solitons Fractals 116, 136-145 (2018)

29. Aljoudi, S., Ahmad, B., Nieto, J.J., Alsaedi, A.: A coupled system of Hadamard type sequential fractional differential equations with coupled strip conditions. Chaos Solitons Fractals 91, 39-46 (2016)

30. Alsaedi, A., Ntouyas, S.K., Agarwal, R.P., Ahmad, B.: On Caputo type sequential fractional differential equations with nonlocal integral boundary conditions. Adv. Differ. Equ. 2015, 33 (2015)

31. Abbas, S., Benchohra, M., Lazreg, J., Nieto, J.J.: On a coupled system of Hilfer-Hadamard fractional differential equations in Banach spaces. J. Nonlinear Funct. Anal. 2018, Article ID 12 (2018)

32. Abbas, S., Benchohra, M., Hamidi, N., Zhou, Y.: Implicit coupled Hilfer-Hadamard fractional differential systems under weak topologies. Adv. Differ. Equ. 2018, 328 (2018)

33. Hilfer, R.: Threefold introduction to fractional derivatives. In: Anomalous Transport: Foundations and Applications, pp. 17-73 (2008)

34. Qassim, M.D., Furati, K.M., Tatar, N.-E.: On a differential equation involving Hilfer-Hadamard fractional derivative. Abstr Appl. Anal. 2012, Article ID 391062 (2015). https://doi.org/10.1155/2012/391062

35. Gambo, Y.Y., Jarad, F., Baleanu, D., Abdeljawad, T.: On Caputo modification of the Hadamard fractional derivatives. Adv. Differ. Equ. 2014, 10 (2014)

36. Granas, A., Dugundji, J.: Fixed Point Theory. Springer, New York (2005)

37. Deimling, K.: Nonlinear Functional Analysis. Springer, New York (1985) 